\title{
Effects of anagliptin on the stress induced accelerated senescence of human umbilical vein endothelial cells
}

\author{
Seon Mee Kang ${ }^{1,2} \wedge$, Hye Sook Jung ${ }^{2} \wedge$, Min Jeong Kwon ${ }^{\wedge}$, Soon Hee Lee ${ }^{1 \wedge}$, Jeong Hyun Park ${ }^{1,2} \wedge$ \\ ${ }^{1}$ Department of Internal Medicine, Busan Paik Hospital, College of Medicine, Inje University, Busan, South Korea; ${ }^{2}$ Paik Institute for Clinical \\ Research, Inje University, Busan, South Korea \\ Contributions: (I) Conception and design: SM Kang, HS Jung, JH Park; (II) Administrative support: None; (III) Provision study materials or patients: \\ SM Kang, HS Jung; (IV) Collection and assembly of data: SM Kang, HS Jung; (V) Data analysis and interpretations: All authors; (VI) Manuscript \\ writing: All authors; (VII) Final approval of manuscript: All authors. \\ Correspondence to: Jeong Hyun Park. Department of Internal Medicine, Busan Paik Hospital, College of Medicine, Inje University, 75 Bokji-ro, \\ Busanjin-gu, Busan, 47392, Republic of Korea. Email: pjhdoc@chol.com; Soon Hee Lee. Department of Internal Medicine, Busan Paik Hospital, \\ College of Medicine, Inje University, 75 Bokji-ro, Busanjin-gu, Busan, 47392, Republic of Korea. Email: 68shlee@hanmail.net.
}

Background: Dipeptidyl peptidase 4 (DPP-4) inhibitors have been used to treat type 2 diabetes mellitus (T2DM) via inhibition of the enzymatic activity of DPP-4 in degrading active circulating glucagon-like peptide-1. In addition to their glucose-lowering effect, DPP-4 inhibitors have pleiotropic effects. Cellular senescence regarded as important pathophysiological mechanism underlying many degenerative diseases, including atherosclerosis. This study was performed to examine whether the DPP-4 inhibitor, anagliptin, can directly protect against stress-induced accelerated senescence (SIAS) of vascular endothelial cells, regardless of changes in ambient glucose level.

Methods: Cultured human umbilical vein endothelial cells (HUVECs) were exposed to various concentrations of $\mathrm{H}_{2} \mathrm{O}_{2}$, and a fixed high concentration of glucose $(25 \mathrm{mM})$ with varying concentrations of palmitate. Changes in cell viability, senescence-associated beta-galactosidase (SA- $\beta-G a l)$, p16 protein, markers of endoplasmic reticulum (ER) stress, NOX4, NLRP inflammasome, lactate dehydrogenase (LDH) release and interleukin (IL) $1 \beta$ levels were measured by Cell Counting Kit- 8 assay, immunofluorescent staining, Western blotting, and enzyme-linked immunosorbent assay, respectively before and after application of anagliptin.

Results: The application of oxidative and glucolipotoxic stresses markedly increased the degree of SIAS of HUVECs, represented by increased SA- $\beta-$ Gal immunopositivity and p16 protein expression. Aggravation of ER stress and inflammatory response were also observed through increased levels of ATF4, CHOP, peIF2 $\alpha$, NOX4, NLRP inflammasome, LDH, and IL1 $\beta$. These changes were markedly reversed by the administration of anagliptin.

Conclusions: The DPP-4 inhibitor anagliptin effectively protects HUVECs against SIAS, suggesting its potential use in the development of new treatment strategies for aging.

Keywords: Human umbilical vein endothelial cell (HUVEC); oxidative stress; glucolipotoxicity; senescence; anagliptin

Submitted Jan 26, 2021. Accepted for publication Mar 14, 2021.

doi: $10.21037 /$ atm-21-393

View this article at: http://dx.doi.org/10.21037/atm-21-393

^ ORCID: Seon Mee Kang, 0000-0002-4056-7525; Hye Sook Jung, 0000-0001-7940-7249; Min Jeong Kwon, 0000-0002-9616-870X; Soon Hee Lee, 0000-0003-4776-0501; Jeong Hyun Park, 0000-0002-0045-4438. 


\section{Introduction}

Type 2 diabetes mellitus (T2DM) is associated with a twofold increase in risk of cardiovascular disease (CVD), such as myocardial infarction, stroke, and peripheral vascular disease, and CVD is the most common cause of death in patients with T2DM (1). Numerous lines of evidence support the major role of endothelial dysfunction defined as a condition of altered metabolism and function of the endothelium and resulting defective repair of vascular injury, in the development of diabetic micro- and macrovascular complications $(2,3)$. The main mechanism of endothelial dysfunction in T2DM is elevated generation of reactive oxygen species (ROS) induced by hyperglycemia and conditions associated with diabetes, such as hypertension and dyslipidemia (4). In addition, endothelial cell senescence and apoptosis play key roles in the development of atherosclerosis, sharing a common pathophysiological mechanism of oxidative stress $(5,6)$.

Cellular senescence is characterized by irreversible cell cycle arrest, multiple changes in gene expression, and production of proinflammatory, and matrix-degrading molecules known as the senescent associated secretory phenotype (SASP). Cells can be in a state of stress-induced accelerated senescence (SIAS), which results from DNA damage in response to elevated oxidative stress (7). There is accumulating evidence for the potential involvement of cellular senescence in many aging and age-related disorders, such as CVD. Senescent vascular endothelial cells accelerate atheroma progression and vascular disease development via the accumulation of oxidized low-density lipoprotein (LDL), inflammation of vascular smooth muscle cells, monocyte chemotaxis, and consequent foam cell formation (8).

Dipeptidyl peptidase 4 (DPP-4) is a regulatory serine protease that is widely distributed in tissues as both soluble and membrane-bound glycoproteins (9). Inhibition of DPP-4 enzymatic activity is a glucose-lowering treatment for T2DM. The classical mechanism of action of DPP4 inhibitors is inhibition DPP-4 enzyme activity, which prevents inactivation of the gut-derived incretin hormone, glucagon-like peptide 1 (GLP-1), in the peripheral circulation (10). Increased circulating active GLP-1 stimulates insulin secretion and inhibits glucagon secretion, resulting in reduced postprandial and fasting glucose, and hemoglobin A1c (HbA1c) levels. Studies have suggested that DPP-4 inhibitors exert diverse nonclassical actions in addition to the glucose-lowering effect, and they exhibit protective effects against diabetic complications affecting the heart, kidneys, retina, neurons, and liver (11). Recent studies showed that DPP-4 inhibition with several DPP4 inhibitors delayed the progression of premature aging and vascular endothelial senescence in both in- vivo and vitro models (12-14). Moreover, the newly developed DPP4 inhibitor, anagliptin, decreased the level of serum fatty acid-binding protein 4, which is a novel adipokine and is associated with atherosclerosis, in patients with T2DM at high risk for CVD regardless of HbA1c or LDL cholesterol levels (15). However, the molecular basis of the action of anagliptin in vascular protection is not clear. In particular, there have been few studies regarding the molecular mechanisms of the action of anagliptin related to cellular senescence. This study was performed to evaluate whether the DPP-4 inhibitor, anagliptin, can protect vascular endothelial cells against SIAS independent of changes in ambient glucose level. We present the following article in accordance with the MDAR reporting checklist (available at http://dx.doi.org/10.21037/atm-21-393).

\section{Methods}

\section{Cell culture and experimental design}

Human umbilical vein endothelial cells (HUVECs) were purchased from PromoCell (MO, USA), and maintained at $37{ }^{\circ} \mathrm{C}$ under a humidified atmosphere of $95 \%$ air and $5 \%$ $\mathrm{CO}_{2}$ in endothelial growth medium containing $2 \%$ fetal bovine serum. HUVECs between the third and seventh passage were used in all experiments. The cells were seeded at a density of $7 \times 10^{3}$ cells/well in 96 -well plates and incubated for 24 hours. The cells were then incubated in the presence of one of three concentrations of $\mathrm{H}_{2} \mathrm{O}_{2}(100,200$, or $300 \mu \mathrm{M}$ ) for 48 hours. In addition, the cells were exposed to high glucose concentration $(25 \mathrm{mM})$ along with one of four concentrations of palmitate $(200,300,400$, or $500 \mu \mathrm{M})$ for 72 hours. Palmitate was prepared through a conjugation reaction with $100 \%$ of methanol to get into solution.

\section{Cell viability and lactate dehydrogenase $(\mathrm{LDH})$ release assay}

Cell viability was measured with the Cell Counting Kit (CCK)-8 assay (Dojindo, Kumamoto, Japan) according to the manufacturer's protocol. The medium in each well of the 96-well plates was replaced with CCK-8 reaction buffer, and the cells were incubated for 2 hours at $37{ }^{\circ} \mathrm{C}$ with $5 \%$ $\mathrm{CO}_{2}$. The optical density was measured at a wavelength of 
$450 \mathrm{~nm}\left(\mathrm{OD}_{450}\right)$ was measured using an automatic plate reader. Cell death was determined by LDH release assay, and quantified with commercially available enzyme-linked immunosorbent assay (ELISA) kits (Abcam, MA, USA). The $\mathrm{OD}_{450}$ was measured with a microtiter plate reader.

\section{Senescence-associated beta-galactosidase staining}

Cellular senescence was visualized by senescence-associated beta-galactosidase (SA- $\beta-\mathrm{Gal})$ staining. HUVECs were washed with phosphate-buffered saline (PBS). SA- $\beta-\mathrm{Gal}$ working solution (pH 6.0) (Cell Signaling, MA, USA) was added, and the cells were observed under an inverted microscope after 24 hours.

\section{Immunofluorescent staining}

Immunofluorescent staining was performed to determine the cellular distribution of $\mathrm{p} 16$, and ROS generation. For detection of p16 expression, HUVECs incubated under the experimental conditions described in experimental design were fixed with cytoperm/cytofix (BD, CA, USA) for 15 minutes, and incubated with blocking buffer (5\% serum and $0.3 \%$ Triton $\mathrm{X}-100$ in PBS) for 1 hour. The medium was then replaced with medium containing anti-p16 antibody for 1 hour at room temperature. The mouse-anti Alexa 488 Fluor $^{\circledR}$, secondary antibody, and DAPI $(1 \mu \mathrm{g} / \mathrm{mL})$ were then added. In addition, HUVECs incubated under the experimental conditions described in experimental design were incubated with Mito SOX (Invitrogen, OR, USA) for 30 minutes at $37{ }^{\circ} \mathrm{C}$ with $5 \%$ $\mathrm{CO}_{2}$ for measurement of ROS generation. An inverted laser confocal microscope (BS-51; Olympus, Tokyo, Japan) was used for staining visualization.

\section{Enzyme-linked immunosorbent assay}

Levels of interleukin (IL)-1 $\beta$, a marker of oxidative stress and inflammation, in the supernatant of cultured HUVECs were measured with commercially available standard sandwich enzyme-linked kits (Abcam, MA, USA) according to the manufacturer's instructions. The $\mathrm{OD}_{450}$ was measured with a microtiter plate reader within 15 minutes.

\section{Western blotting assay}

HUVECs were washed with PBS and lysed with mammalian tissue lysis/extraction reagent including protease inhibitor
(Roche, IN, USA). Protein concentrations were determined using BCA protein assay kit (Pierce Chemicals, CA, USA), and $1 \times$ sodium dodecyl sulfate (SDS) sample buffer $(50 \mathrm{mM}$ Tris, $\mathrm{pH}$ 6.8, 2\% SDS, 10\% glycerol, $50 \mathrm{mM}$ dithiothreitol, and $0.01 \%$ bromophenol blue) was added. Proteins were separated by SDS-polyacrylamide gel electrophoresis in $10-15 \%$ gels, transferred onto polyvinylidene difluoride membranes, and incubated with anti-p16 (ab54210, Abcam, MA, USA), anti-ATF4 (11815, Cell Signaling, MA, USA), anti-peIF2 $\alpha$ (9721, Cell Signaling, MA, USA), antiNOX4 (LS-C313066, Lifespan Biosciences, WA, USA), anti-NLRP3 (ab214185, Abcam, MA, USA), anti-cleaved caspase-1 (4199, Cell signaling, MA, USA), anti-GAPDH (2118, Cell signaling, MA, USA), and anti-tubulin (3873, Cell signaling, MA, USA), antibodies at $4{ }^{\circ} \mathrm{C}$ overnight. Alkaline phosphatase-conjugated goat anti-rabbit (A120101AP, Bethyl, TX, USA) or anti-mouse secondary antibody (A90-116AP, Bethyl, TX, USA) was applied for 1 hour at room temperature, and membranes were developed using an alkaline phosphatase conjugate substrate kit (Bio-Rad, CA, USA). Developed protein bands were quantified using Image J (NIH, Bethesda, MD, USA).

\section{Statistics}

The results were expressed as the mean \pm standard deviation, and statistical analyses were performed using SPSS software (version 20.0; IBM Corp., NY, USA). Differences between groups were compared by Student's $t$-test. In all analyses, $\mathrm{P}<0.05$ was taken to indicate statistical significance.

\section{Results}

\section{p16 protein expression in HUVECs under stress conditions}

p16 is a biomarker of cellular senescence. First, we measured p16 protein expression levels in HUVECs under various stress conditions induced by $\mathrm{H}_{2} \mathrm{O}_{2}$ or high glucose with palmitate (HGP) (Figure S1A,B). HUVECs were incubated in the presence of one of three concentrations of $\mathrm{H}_{2} \mathrm{O}_{2}(100,200$, or $300 \mu \mathrm{M})$ for 48 hours. In addition, the cells were exposed to a high concentration glucose $(25 \mathrm{mM})$ with one of four concentrations of palmitate $(200,300$, 400 , or $500 \mu \mathrm{M})$ for 72 hours. After fixation and antibody reaction, immunofluorescent staining was performed. p16 protein expression was increased after exposure to 200 and $300 \mu \mathrm{M} \mathrm{H}_{2} \mathrm{O}_{2}$ as well as 400 and $500 \mu \mathrm{M}$ palmitate under 

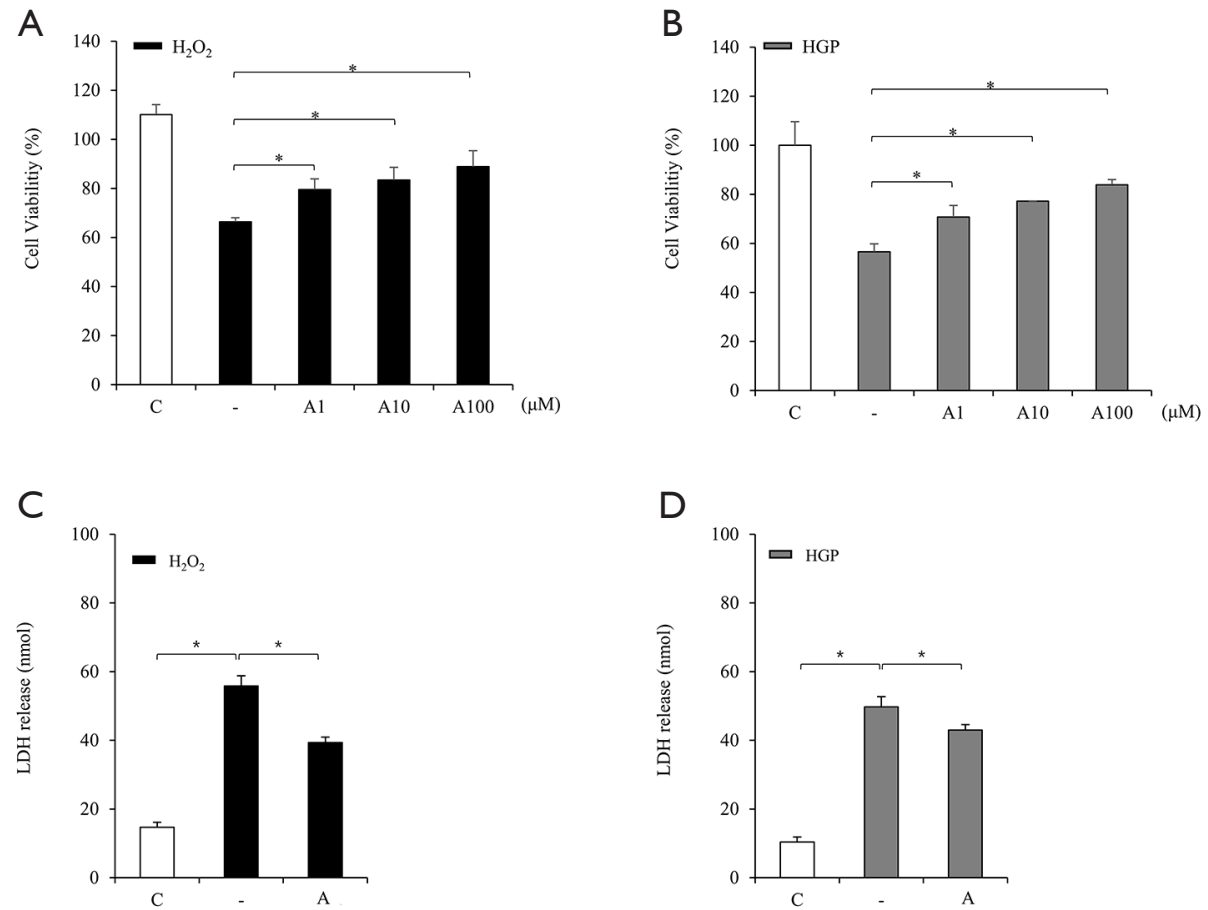

Figure 1 Effects of anagliptin on cell death of HUVECs. (A) Cell viability measured using the CCK-8 assay was decreased to $66 \%$ by oxidative stress induced by $200 \mu \mathrm{M}$ of $\mathrm{H}_{2} \mathrm{O}_{2}$ which was attenuated in a dose-dependent manner to $79 \%, 83 \%$, and $89 \%$ by treatment with 1,10 , and $100 \mu \mathrm{M}$, respectively. (B) Cell viability measured using CCK-8 assay was decreased to $56 \%$ by HGP (25 mM glucose and 400 $\mu \mathrm{M}$ palmitate), which was attenuated in a dose dependent manner to $71 \%, 77 \%$, and $84 \%$ by treatment with 1,10 , and $100 \mu \mathrm{M}$ anagliptin, respectively. (C,D) LDH release, indicating cellular death, was significantly increased after $\mathrm{H}_{2} \mathrm{O}_{2}$ and HGP treatment, and reduced by anagliptin treatment. ${ }^{*} \mathrm{P}<0.05$. C, control; A, anagliptin.

conditions of hyperglycemia (HGP), respectively. These findings showed that HUVECs were induced enter a state of SIAS by oxidative stress or glucolipotoxicity in a dosedependent manner. Based on these findings, we decided to use the concentration of $200 \mu \mathrm{M} \mathrm{H}_{2} \mathrm{O}_{2}$, and $25 \mathrm{mM}$ glucose and $400 \mu \mathrm{M}$ palmitate as oxidative stress condition, and HGP stress condition exposure to HUVECs, respectively.

\section{Anagliptin attenuated cell death of HUVECs under stress conditions}

To test the protective effect of anagliptin against cell death induced by $\mathrm{H}_{2} \mathrm{O}_{2}$ or glucolipotoxicity, cell viability was measured at various concentrations of anagliptin using the CCK-8 assay (Figure 1A,B). Anagliptin was contributed from Sanwa Kagaku Kenkyusho Co., Ltd. (Nagoya, Japan). Anagliptin was prepared as a solution by dissolving with $100 \%$ of DMSO. Cell viability was decreased to $66 \%$ by $200 \mu \mathrm{M} \mathrm{H}_{2} \mathrm{O}_{2}$ treatment, which was attenuated to $79 \%$,
$83 \%$, and $89 \%$ by treatment with 1,10 , and $100 \mu \mathrm{M}$ anagliptin, respectively (Figure $1 A$ ). Cell viability was decreased to $56 \%$ after exposure to HGP ( $25 \mathrm{mM}$ glucose and $400 \mu \mathrm{M}$ palmitate) treatment, which was attenuated to $71 \%, 77 \%$, and $84 \%$ by treatment with 1,10 , and $100 \mu \mathrm{M}$ anagliptin, respectively (Figure $1 B$ ) The potent protective effect of anagliptin on HUVECs was dose dependent. In addition, $\mathrm{H}_{2} \mathrm{O}_{2}$ - or HGP-treated cells showed markedly increased of release of $\mathrm{LDH}$ into the medium compared to controls, and anagliptin treatment significantly reduced LDH release (Figure 1C,D).

\section{Anagliptin delayed cellular senescence of HUVECs under stress conditions}

To determine whether anagliptin can prevent the SIAS of HUVECs, we analyzed p16 protein expression and staining for SA- $\beta-G a l$, which are regarded as biomarkers of cellular senescence (Figure $2 A, B, C, D$ ). p16 protein expression was 

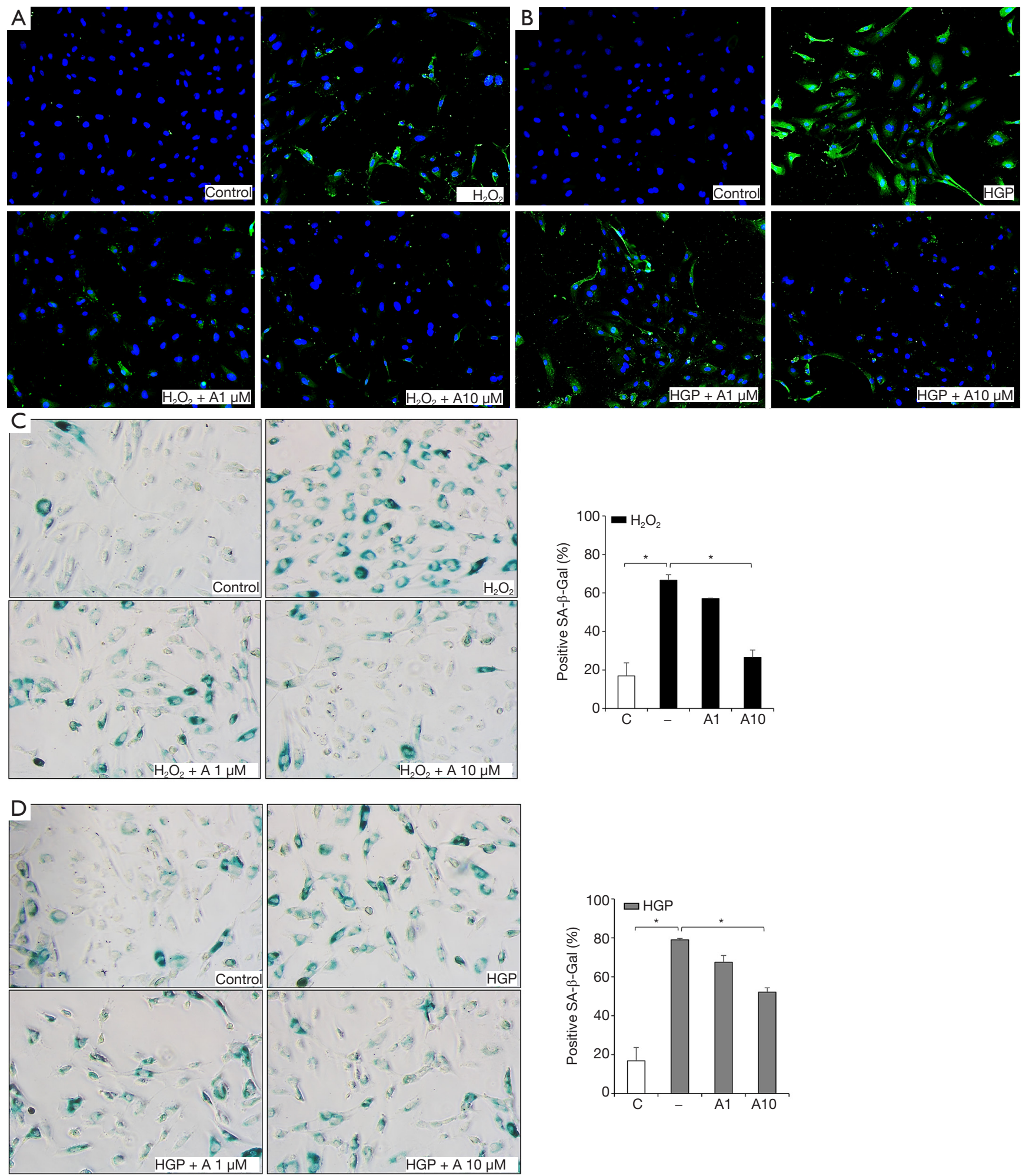
$E$
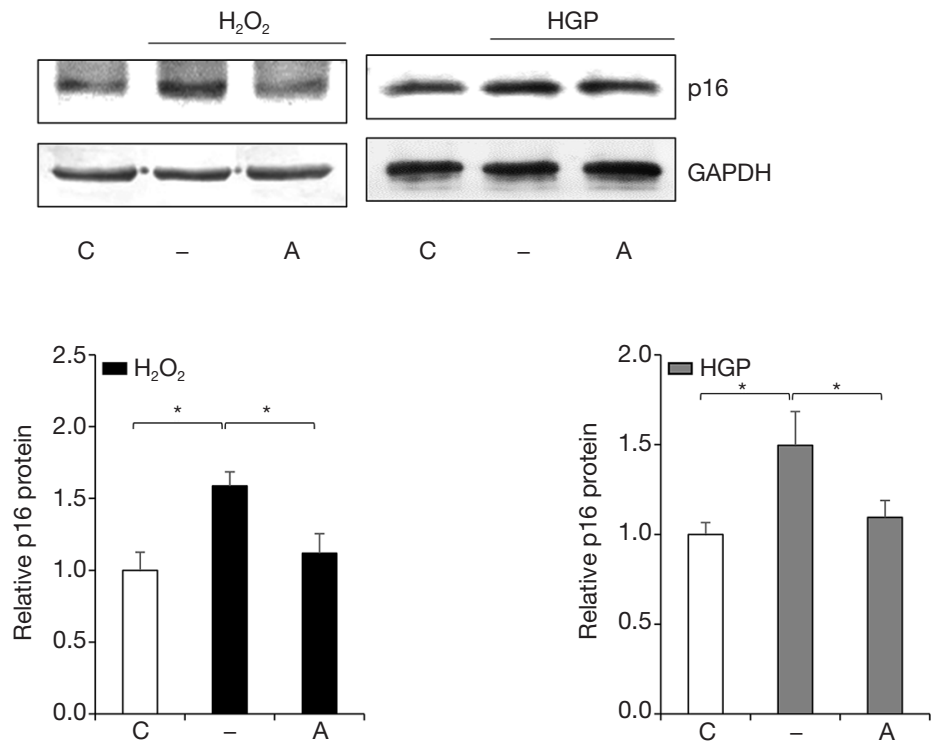

Figure 2 Effects of anagliptin on cellular senescence of HUVECs. (A,B) p16 protein expression (green) detected by immunofluorescent staining was increased after $\mathrm{H}_{2} \mathrm{O}_{2}$ or HGP treatment, and decreased significantly after treatment with 1 and $10 \mu \mathrm{M}$ anagliptin, respectively. (C,D) Senescent (SA- $\beta$-Gal-positive, green) cells increased after $\mathrm{H}_{2} \mathrm{O}_{2}$ or HGP treatment, and decreased after treatment with 1 and 10 $\mu \mathrm{M}$ anagliptin, respectively. (E) Western blotting analysis of p16 protein levels in HUVECs. ${ }^{*} \mathrm{P}<0.05$. C, control; A, anagliptin. GAPDH, protein loading control.

increased by $\mathrm{H}_{2} \mathrm{O}_{2}$ treatment, and reduced by treatment with 1 and $10 \mu \mathrm{M}$ anagliptin, in a dose- dependent manner (Figure 2A). Similar patterns of changes were also seen under conditions of HGP stress (Figure 2B). The proportion of SA$\beta$-Gal-positive cells was significantly increased in the $\mathrm{H}_{2} \mathrm{O}_{2}$ treatment group, and decreased with 1 and $10 \mu \mathrm{M}$ anagliptin treatment (Figure 2C). The same patterns of results were observed with HGP stress and anagliptin treatment, and the changes were also dose dependent (Figure 2D). However, the extent of changes was greater with $\mathrm{H}_{2} \mathrm{O}_{2}$-induced oxidative stress. In addition, Western blotting analysis for $\mathrm{p} 16$ protein expression showed identical results (Figure 2E).

\section{Anagliptin treatment attenuated endoplasmic reticulum (ER) stress markers in HUVECs under stress conditions}

To examine whether the induction of cell death of HUVECs cultured under conditions of oxidative or HGP stress was associated with ER stress, and whether anagliptin could reduce the levels of ER stress markers, we analyzed the expression of the ER stress markers, activating transcription factor 4 (ATF4), C/EBP homologous protein (CHOP), and phosphor-eukaryotic initiation factor $2 \alpha$ (peIF $2 \alpha$ ) by Western blotting assay (Figure $3 A, B$ ). The levels of CHOP and peIF $2 \alpha$ expression were increased with $\mathrm{H}_{2} \mathrm{O}_{2}$ treatment and reduced by anagliptin, while the level of ATF4 showed no changes with either $\mathrm{H}_{2} \mathrm{O}_{2}$, and anagliptin treatment (Figure 3A). All three markers were increased by HGP treatment, and attenuated by anagliptin (Figure 3B).

\section{Anagliptin treatment decreased ROS generation in HUVECs under stress conditions}

To examine whether anagliptin could reduce the generation of cellular ROS induced by $\mathrm{H}_{2} \mathrm{O}_{2}$ or HGP, we measured ROS and NADPH oxidase 4 (NOX4) expression in HUVECs (Figure 4A,B,C). As expected, significant increases in ROS levels were observed in cells exposed to $\mathrm{H}_{2} \mathrm{O}_{2}$ or HGP, and anagliptin significantly decreased ROS generation in a dose- dependent manner (Figure $4 A, B$ ). The level of NOX4, an enzyme involved in ROS production, was increased four folds with $\mathrm{H}_{2} \mathrm{O}_{2}$ and HGP treatment compared to controls, but was attenuated to a level similar to that of the control group after anagliptin treatment 
A

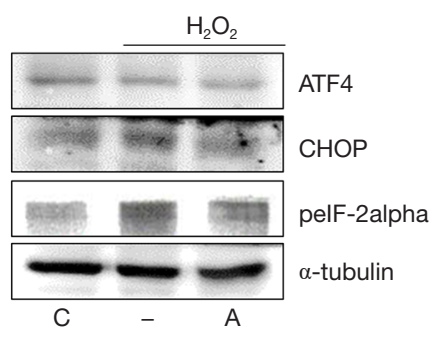

B

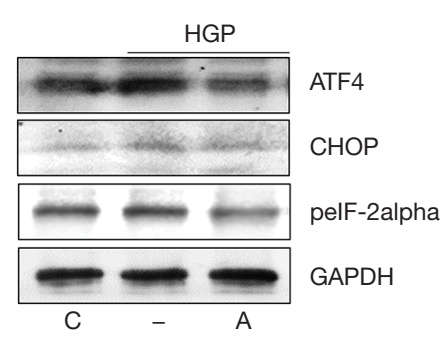

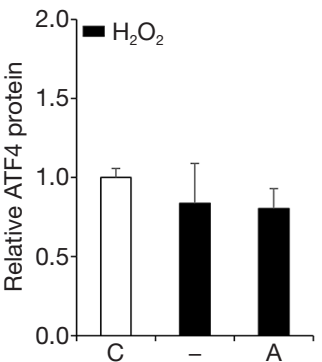
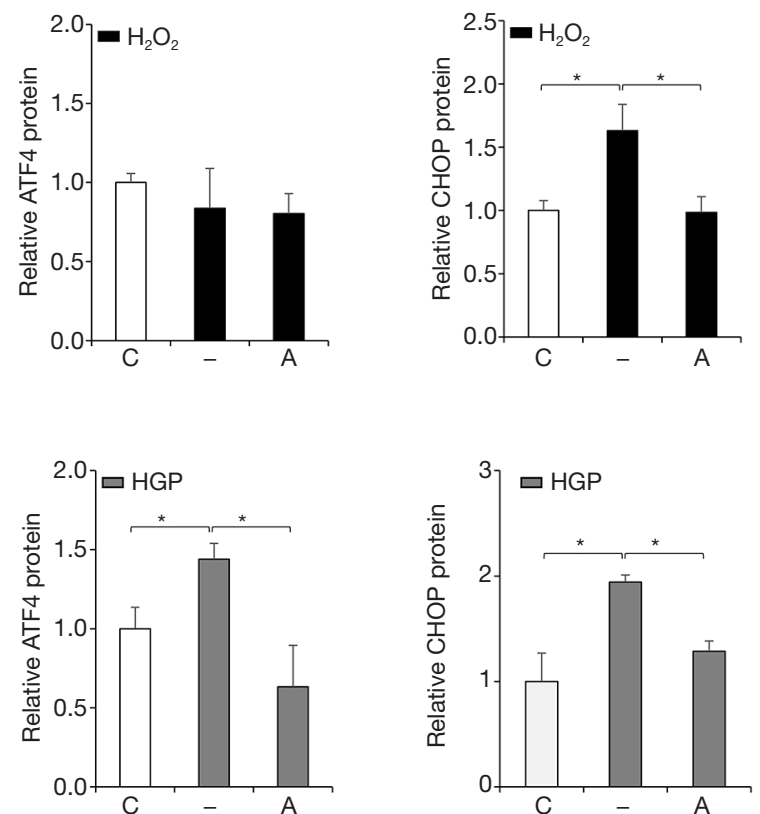
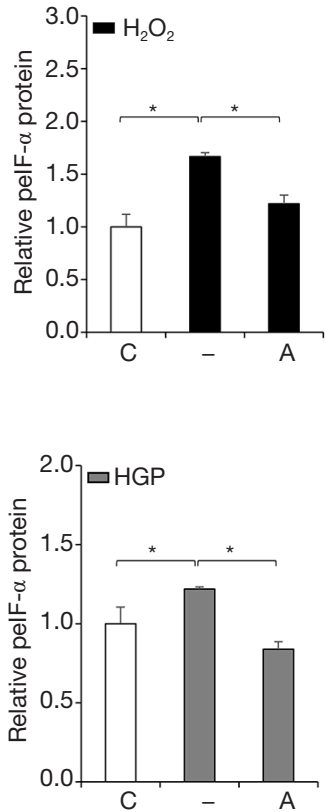

Figure 3 Effects of anagliptin on ER stress markers of HUVECs under stress conditions. (A,B) Western blotting analysis of ATF4, CHOP, and peIF2 $\alpha$ after $\mathrm{H}_{2} \mathrm{O}_{2}$ and HGP treatment, respectively. There was no change in ATF4 level under $\mathrm{H}_{2} \mathrm{O}_{2}$ treatment; however, all other markers increased under both stress conditions and decreased with anagliptin treatment. C, control; A, anagliptin; GAPDH, protein loading control. ${ }^{*} \mathrm{P}<0.05$.

(Figure 4C).

\section{Anagliptin treatment decreased markers related to inflammasome activation in HUVECs under stress conditions}

Inflammation is an important factor contributing to atherosclerosis. The nucleotide-binding oligomerization domain-like receptor protein 3 (NLRP3) inflammasome pathway is an important driver of atherosclerotic CVD (16). We investigated NLRP3 inflammasome activation in HUVECs by $\mathrm{H}_{2} \mathrm{O}_{2}$ or HGP, and whether anagliptin could reduce the activation of this inflammatory pathway (Figure $5 A, B, C, D$ ). NLRP promotes IL- $1 \beta$ induction via the cleavage of caspase- 1 . As expected, the levels of NLRP and cleaved caspase- 1 were increased by 2 - and 1.5 -fold compared to the control group by $\mathrm{H}_{2} \mathrm{O}_{2}$ treatment, respectively, and were significantly attenuated to levels close to those of the control group after anagliptin treatment (Figure $5 A$ ). Similar patterns of responses were observed in the HGP stress group (Figure 5B). ELISA showed that anagliptin treatment attenuated the increased in IL$1 \beta$ expression induced by $\mathrm{H}_{2} \mathrm{O}_{2}$ oxidative or HGP metabolic stress, respectively (Figure 5C,D). These findings suggest that anagliptin could have inhibitory effects on NLRP3 inflammasome pathway activation and downstream protein expression.

\section{Discussion}

Since the discovery of long-lived strains of Caenorhabditis elegans in 1934 (17), ageing research has emerged as an important field. Cellular senescence classically is defined by irreversible cell cycle arrest that is activated by multiple stressors, such as DNA damage, oxidative stress, oncogenic insults or cytotoxic agents. These stimuli trigger various signaling pathways, many of which activate p16, p21, and $\mathrm{p} 53$, and eventually activate cell cycle inhibitors and tumor suppressors (18). As they age, cells develop a proinflammatory phenotype and recruit immune cells through the SASP. Morphologically, senescent cells are usually bulky and SA- $\beta-$ Gal activity due to mitochondrial dysfunction and lysosome biogenesis (19). Therefore, p16 expression and SA- $\beta-$ Gal can be used as biomarkers of cellular senescence. We showed increased p16 expression in HUVECs by immunofluorescent staining under various concentrations of $\mathrm{H}_{2} \mathrm{O}_{2}$-induced oxidative stress or HGP-induced metabolic 

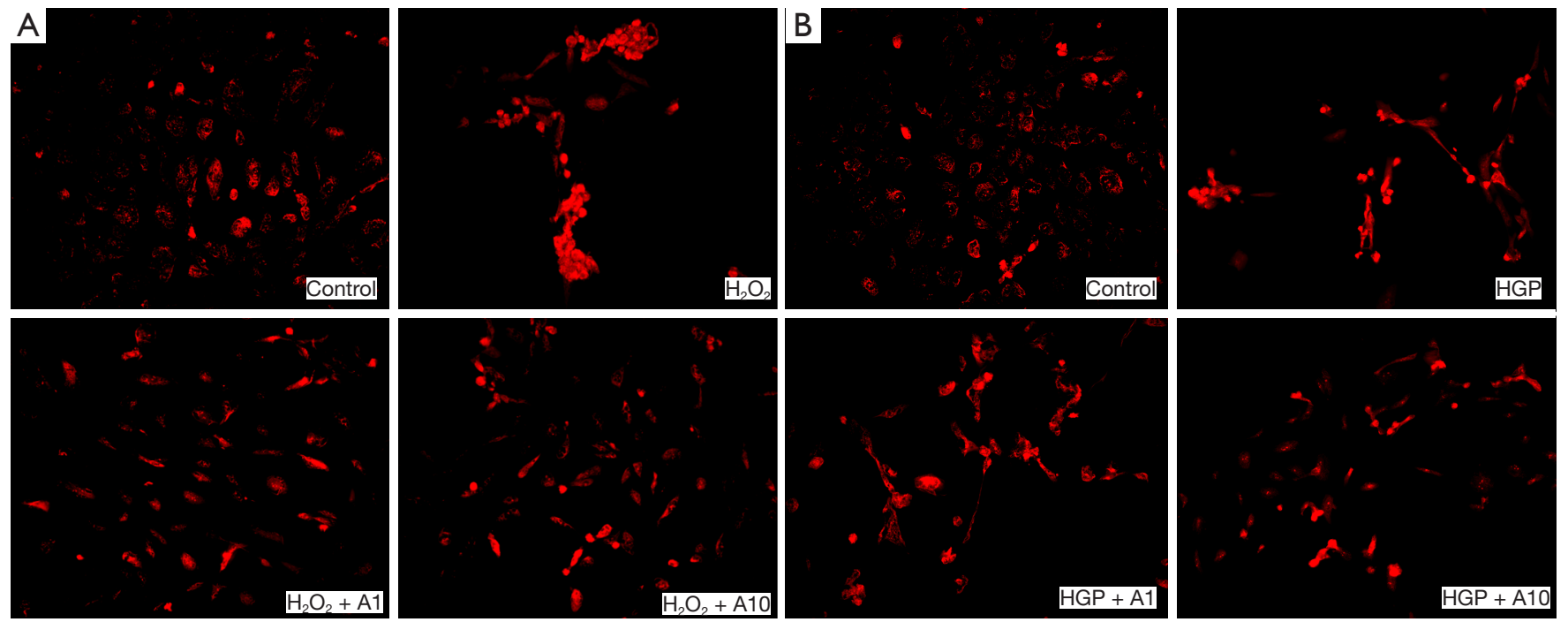

C
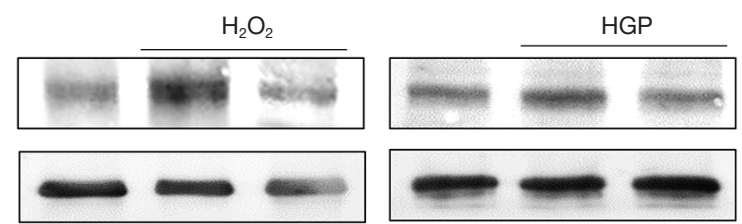

NOX-4

C

A

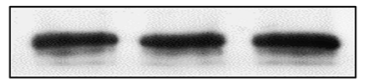

GAPDH
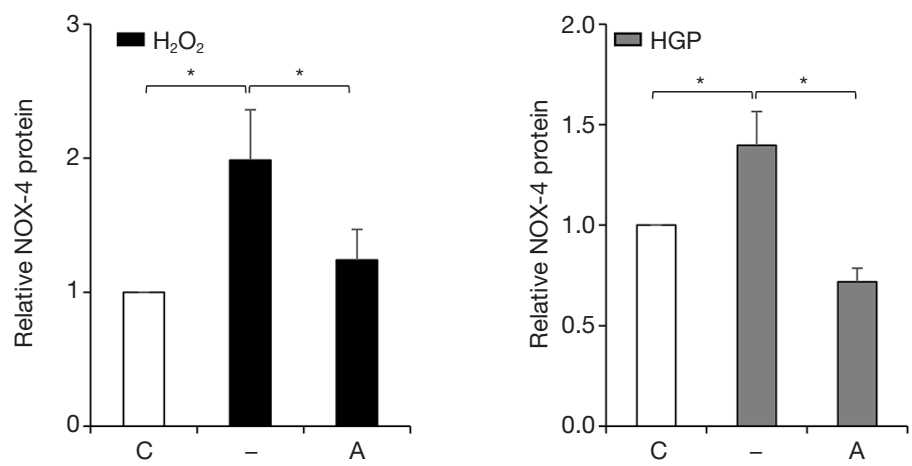

Figure 4 Effects of anagliptin on ROS generation by HUVECs under stress conditions. (A,B) Representative fluorescent ROS measurement (red) in HUVECs after $\mathrm{H}_{2} \mathrm{O}_{2}$ and HGP treatment, respectively. (C) Western blotting assay of NOX4 in HUVECs. The bar graphs show the relative protein expression of NOX4 from HUVECs after $\mathrm{H}_{2} \mathrm{O}_{2}$ and HGP treatment. C, control; A, anagliptin; GAPDH, protein loading control.

stress conditions resembling SIAS, as well as their recovery after anagliptin treatment. In addition, SA- $\beta-$ Gal- stained HUVECs were increased under condition of $\mathrm{H}_{2} \mathrm{O}_{2}$-induced oxidative stress or HGP-induced metabolic stress, which decreased after anagliptin treatment. Taken together, these observations suggest that anagliptin protects HUVECs from SIAS.
In addition, we measured ER stress markers, such as ATF4, CHOP, and peIF2 $\alpha$, which were increased by $\mathrm{H}_{2} \mathrm{O}_{2}$ or HGP treatment, and attenuated by anagliptin treatment. Dysregulation of proteostasis, i.e., of protein production resulting in intracellular accumulation of abnormal protein aggregates, is a prominent feature of age-related disorders (20). Under conditions of ER stress, the unfolded protein response 
A

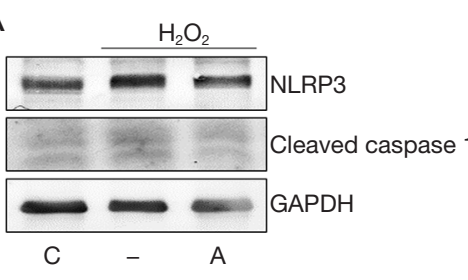

B

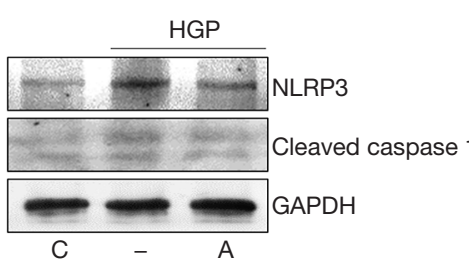

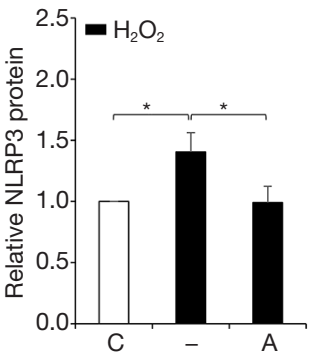

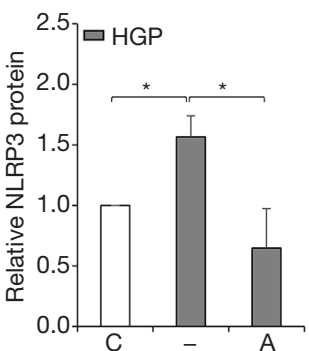

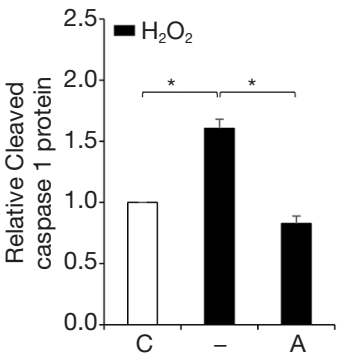
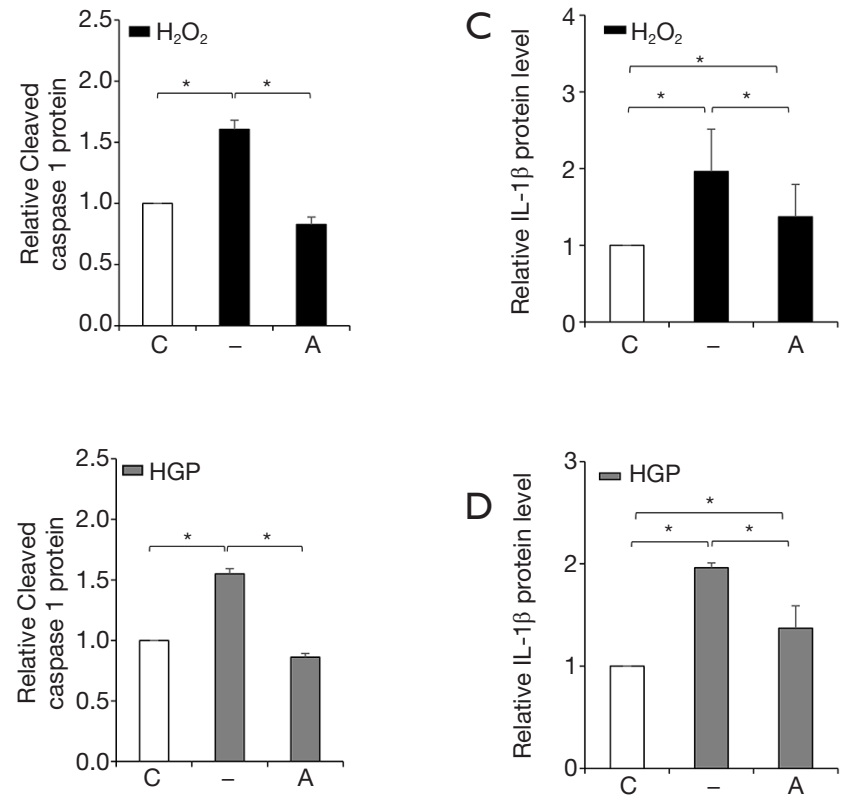

Figure 5 Effects of anagliptin on markers related to inflammasome activation in HUVECs under stress conditions. (A,B) Representative results of western blotting analysis of NLRP and cleaved caspase-1 levels in HUVECs after $\mathrm{H}_{2} \mathrm{O}_{2}$ and HGP treatment, respectively. The bar graphs show the relative protein expression of IL- $1 \beta$ in HUVECs after (C) $\mathrm{H}_{2} \mathrm{O}_{2}$ and (D) HGP treatment. ${ }^{*} \mathrm{P}<0.05$. C, control; A, anagliptin; GAPDH, protein loading control.

(UPR) pathways are activated, and restore the proper protein folding capacity of the cell or trigger apoptosis of damaged cells. That is, ER stress accompanies senescence, and the UPR is a component of the aging process $(21,22)$. In our experiments, the decreased cell viability and increased LDH release of $\mathrm{H}_{2} \mathrm{O}_{2}$ - or HGP-treated HUVECs recovered after anagliptin treatment. These findings present possibility that the protective effects of anagliptin against ER stress partly contribute to its anti-senescence effects and additional study is needed in the future.

Chronic inflammation, particularly the NLRP3 inflammasome pathway, plays an important role in the initiation of atherosclerosis. Inflammasomes are complexes of proteins responsible for a signaling cascade that mediates innate inflammatory responses from NLRP 3 to IL- $1 \beta$ and IL18 via cleavage of caspase-1 (23). The main stimuli of NLRP3 activation are ATP, oxidized LDL, and cholesterol crystals. In addition, the key mechanisms of this process are mitochondrial ROS production, potassium efflux, and lysosomal damage (23-25). Furthermore, accumulating evidence supports the roles of prolonged ER stress and subsequent UPR- related ROS generation in NLRP3 inflammasome activation and atherosclerosis progression (26). In the present study, ROS generation and related NOX4 increased in $\mathrm{H}_{2} \mathrm{O}_{2}$ - or HGP- treated HUVECs and decreased markedly after anagliptin treatment. In addition, markers related to inflammasome activation, NLRP3, cleaved caspase-1, and IL-1 $\beta$ were increased after $\mathrm{H}_{2} \mathrm{O}_{2}$ or HGP treatment and reduced by anagliptin treatment. Taken together, these observations suggest that anagliptin treatment may protect HUVECs from NLRP3 inflammasome activation by reducing mitochondrial ROS generation. In a previous study with $\mathrm{klotho}^{-/-}$mice, which exhibit multiple phenotypes resembling human premature aging, DPP-4 inhibition by linagliptin ameliorated hippocampal neurodegeneration and cognitive impairment via improvement of cerebral blood flow and cerebral endothelial nitric oxide synthase (eNOS) level (12). In another study, Cheng et al. reported the preventive effect of anagliptin against vascular aging of mice under conditions of immobilizationinduced chronic stress. They demonstrated reduced oxidative stress and improved inflammation after anagliptin treatment in the mouse vasculature and in HUVECs (14). A recent study revealed that DPP-4 inhibition with saxagliptin delayed endothelial senescence of rats and reduced oxidative stress and inflammation of HUVECs by regulating the AMPK/SIRT1/ Nrf2 pathway (13).

Atherosclerotic CVD is one of the leading causes of death in diabetic patients. Despite major efforts, intensive glycemic 
control did not show beneficial effects for macrovascular complications but rather excessive glycemic control was shown to be harmful in older patients with longer duration of diabetes combined with CVD (27). Therefore, management of multiple risk factors is emphasized over glucose control alone (28). Indeed, CVD is an age-related disease rather than a complication of T2DM, and is the most common cause of death in the elderly (29). Therefore, senolytics, bioactive compounds that selectively target and induce death of senescent cells, are emerging as a new treatment modality for CVD (30). A previous study showed that DPP-4 expression was selectively increased on the surface of senescent human diploid fibroblasts, using anti-DPP-4 antibody, these senescent cells were isolated by flow cytometry and could be preferentially eliminated (31). In the present study, the antisenescent effect of anagliptin on HUVECs was observed even in cells still in the high-glucose environment. This suggests that antioxidative and anti-senescent effects of anagliptin are not related to the classical DPP-4 inhibitory and glucoselowering mechanism.

This study had several limitations. First, we did not use normal physiological dose of anagliptin in the experiments. Second, there are clear difference between senescence and SIAS. Although p16 protein and SA- $\beta-$ Gal staining are regarded as biomarkers of senescence, they could be elevated under other conditions, such as inflammation. Third, we used only HUVECs in the present study. However, atherosclerotic plaque builds up in arteries and produced by the interaction of vascular endothelial cells, vascular smooth muscle cells, and inflammatory molecules (32).

In conclusion, senescence and anti-senescence process is at least partially involved in the development of diabetic complications or progression of CVD. DPP-4 inhibition with anagliptin improved ER stress, decreased oxidative stress and inflammation, and finally ameliorated the SIAS of HUVECs regardless of glucose level. These findings suggest that antisenescence mechanisms of DPP-4 inhibitors can be used in the development of new treatment strategies for aging.

\section{Acknowledgments}

Funding: This work was supported by the Basic Science Research Program funded by JW Joong Wae Pharmaceutical.

\section{Footnote}

Reporting Checklist: The authors have completed the MDAR reporting checklist. Available at http://dx.doi.org/10.21037/ atm-21-393

Data Sharing Statement: Available at http://dx.doi. org/10.21037/atm-21-393

Peer Review File: Available at http://dx.doi.org/10.21037/ atm-21-393

Conflicts of Interest: All authors have completed the ICMJE uniform disclosure form (available at http://dx.doi. org/10.21037/atm-21-393). All authors report that this study was funded by JW Pharmaceutical.

Ethical Statement: The authors are accountable for all aspects of the work in ensuring that questions related to the accuracy or integrity of any part of the work are appropriately investigated and resolved.

Open Access Statement: This is an Open Access article distributed in accordance with the Creative Commons Attribution-NonCommercial-NoDerivs 4.0 International License (CC BY-NC-ND 4.0), which permits the noncommercial replication and distribution of the article with the strict proviso that no changes or edits are made and the original work is properly cited (including links to both the formal publication through the relevant DOI and the license). See: https://creativecommons.org/licenses/by-nc-nd/4.0/.

\section{References}

1. Morrish NJ, Wang SL, Stevens LK, et al. Mortality and causes of death in the WHO Multinational Study of Vascular Disease in Diabetes. Diabetologia 2001;44 Suppl 2:S14-21.

2. Anderson TJ. Nitric oxide, atherosclerosis and the clinical relevance of endothelial dysfunction. Heart Fail Rev 2003;8:71-86.

3. Shi Y, Vanhoutte PM. Macro- and microvascular endothelial dysfunction in diabetes. J Diabetes 2017;9:434-49.

4. Kim JA, Montagnani M, Koh KK, et al. Reciprocal relationships between insulin resistance and endothelial dysfunction: molecular and pathophysiological mechanisms. Circulation 2006;113:1888-904.

5. Erusalimsky JD. Vascular endothelial senescence: from mechanisms to pathophysiology. J Appl Physiol (1985) 2009;106:326-32.

6. Hayashi T, Matsui-Hirai H, Miyazaki-Akita A, et al. 
Endothelial cellular senescence is inhibited by nitric oxide: implications in atherosclerosis associated with menopause and diabetes. Proc Natl Acad Sci U S A 2006;103:17018-23.

7. Childs BG, Durik M, Baker DJ, et al. Cellular senescence in aging and age-related disease: from mechanisms to therapy. Nat Med 2015;21:1424-35.

8. Machado-Oliveira G, Ramos C, Marques ARA, et al. Cell Senescence, Multiple Organelle Dysfunction and Atherosclerosis. Cells 2020;9:2146.

9. Flatt PR, Bailey CJ, Green BD. Dipeptidyl peptidase IV (DPP IV) and related molecules in type 2 diabetes. Front Biosci 2008;13:3648-60.

10. Ahren B. Dipeptidyl peptidase-4 inhibitors: clinical data and clinical implications. Diabetes Care 2007;30:1344-50.

11. Bae EJ. DPP-4 inhibitors in diabetic complications: role of DPP-4 beyond glucose control. Arch Pharm Res 2016;39:1114-28.

12. Hasegawa Y, Hayashi K, Takemoto Y, et al. DPP-4 inhibition with linagliptin ameliorates the progression of premature aging in klotho-/- mice. Cardiovasc Diabetol 2017;16:154.

13. Chen Z, Yu J, Fu M, et al. Dipeptidyl peptidase-4 inhibition improves endothelial senescence by activating AMPK/SIRT1/Nrf2 signaling pathway. Biochem Pharmacol 2020;177:113951.

14. Xin M, Jin X, Cui X, et al. Dipeptidyl peptidase-4 inhibition prevents vascular aging in mice under chronic stress: Modulation of oxidative stress and inflammation. Chem Biol Interact 2019;314:108842.

15. Furuhashi M, Sakuma I, Morimoto T, et al. Treatment with anagliptin, a DPP-4 inhibitor, decreases FABP4 concentration in patients with type 2 diabetes mellitus at a high risk for cardiovascular disease who are receiving statin therapy. Cardiovasc Diabetol 2020;19:89.

16. Baragetti A, Catapano AL, Magni P. Multifactorial Activation of NLRP3 Inflammasome: Relevance for a Precision Approach to Atherosclerotic Cardiovascular Risk and Disease. Int J Mol Sci 2020;21:4459.

17. Klass MR. A method for the isolation of longevity mutants in the nematode Caenorhabditis elegans and initial results. Mech Ageing Dev 1983;22:279-86.

18. Munoz-Espin D, Serrano M. Cellular senescence: from physiology to pathology. Nat Rev Mol Cell Biol 2014;15:482-96.

19. Lopez-Otin C, Blasco MA, Partridge L, et al. The hallmarks of aging. Cell 2013;153:1194-217.
20. Kaushik S, Cuervo AM. Proteostasis and aging. Nat Med 2015;21:1406-15.

21. Pluquet O, Pourtier A, Abbadie C. The unfolded protein response and cellular senescence. A review in the theme: cellular mechanisms of endoplasmic reticulum stress signaling in health and disease. Am J Physiol Cell Physiol 2015;308:C415-25.

22. Matos L, Gouveia AM, Almeida H. ER Stress Response in Human Cellular Models of Senescence. J Gerontol A Biol Sci Med Sci 2015;70:924-35.

23. Duewell P, Kono H, Rayner KJ, et al. NLRP3 inflammasomes are required for atherogenesis and activated by cholesterol crystals. Nature 2010;464:1357-61.

24. Man SM, Kanneganti TD. Regulation of inflammasome activation. Immunol Rev 2015;265:6-21.

25. Zhou R, Yazdi AS, Menu P, et al. A role for mitochondria in NLRP3 inflammasome activation. Nature 2011;469:221-5.

26. Tabas I. The role of endoplasmic reticulum stress in the progression of atherosclerosis. Circ Res 2010;107:839-50.

27. Action to Control Cardiovascular Risk in Diabetes Study G, Gerstein HC, Miller ME, et al. Effects of intensive glucose lowering in type 2 diabetes. $\mathrm{N}$ Engl J Med 2008;358:2545-59.

28. Yudkin JS, Richter B, Gale EA. Intensified glucose lowering in type 2 diabetes: time for a reappraisal. Diabetologia 2010;53:2079-85.

29. Robinson JG, Fox KM, Bullano MF, et al. Atherosclerosis profile and incidence of cardiovascular events: a population-based survey. BMC Cardiovasc Disord 2009;9:46.

30. Dookun E, Passos JF, Arthur HM, et al. Therapeutic Potential of Senolytics in Cardiovascular Disease. Cardiovasc Drugs Ther 2020. [Epub ahead of print]. doi: 10.1007/s10557-020-07075-w.

31. Kim KM, Noh JH, Bodogai M, et al. Identification of senescent cell surface targetable protein DPP4. Genes Dev 2017;31:1529-34.

32. Basatemur GL, Jorgensen HF, Clarke MCH, et al. Vascular smooth muscle cells in atherosclerosis. Nat Rev Cardiol 2019;16:727-44.

Cite this article as: Kang SM, Jung HS, Kwon MJ, Lee SH, Park JH. Effects of anagliptin on the stress induced accelerated senescence of human umbilical vein endothelial cells. Ann Transl Med 2021;9(9):750. doi: 10.21037/atm-21-393 

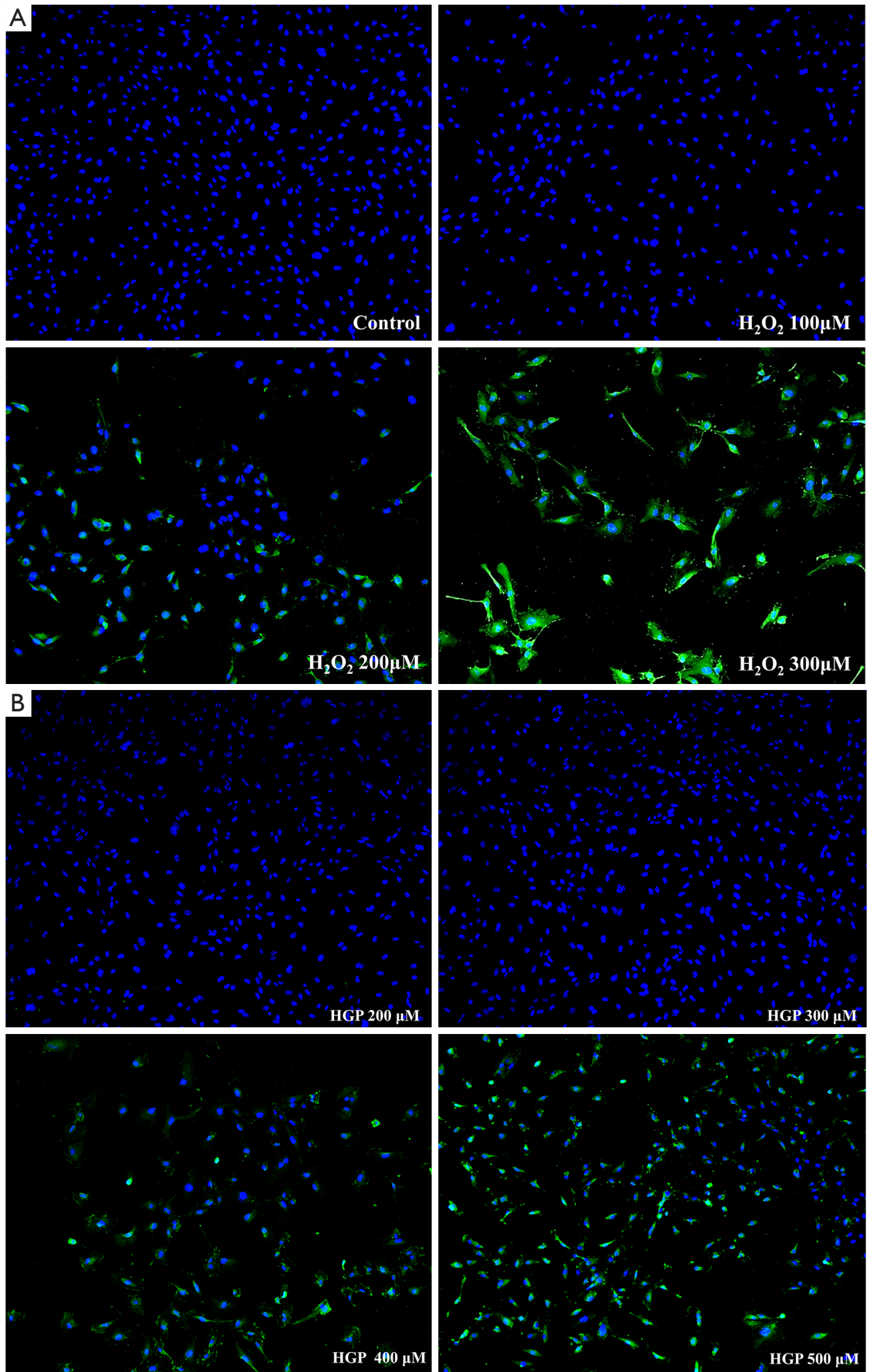

Figure S1 Detection of p16 protein expression in HUVECs under various stress conditions by FISH assay. P16 protein (green), a biomarker of senescence, as increased by 20 and $300 \mu \mathrm{M} \mathrm{H}_{2} \mathrm{O}_{2}$ (A) and 400 and $500 \mu \mathrm{M}$ palmitate with $25 \mathrm{mM}$ glucose (B). 